\title{
RECENT ADVANCES IN TITANIUM TECHNOLOGY IN THE UNITED STATES
}

\author{
Dongjian(Don) $\mathrm{Li}^{1}$, Vasisht Venkatesh ${ }^{2}$ \\ ${ }^{1}$ Arconic, Niles, $\mathrm{OH}$ \\ ${ }^{2}$ Pratt \& Whitney, East Hartford CT
}

\begin{abstract}
$\underline{\text { Abstract }}$
Substantial progress has continued in the US since the last $\left(13^{\text {th }}\right)$ World Titanium Conference with regards to titanium technology, products, processing, computational modeling tools and applications. Titanium components produced by additive manufacturing have been qualified and achieved production applications in commercial and military aircraft hardware. New high-performance titanium alloys have been developed for higher temperature service and applications requiring more demanding static and dynamic properties. Current production processes for titanium have been optimized, and new processes have been developed to further reduce cost and improve product quality. Titanium suppliers, OEM's, government labs and academia are working closely together to address fundamental industry-wide issues. Computer modeling is now used extensively in industrial and research facilities to accelerate the pace and success of these developments.
\end{abstract}

\section{Introduction}

A recent aerospace forecast by the Federal Aviation Administration (FAA) for fiscal years 2019 to 2039 expects air traffic (i.e., number of landings and take offs) to grow from 51.8 million in 2018 to 62 million in 2039. In addition, the U.S. domestic market for the first time has seen 8 consecutive years of passenger growth [1]. Over the next 20-year period 43,000 new commercial aircraft are expected to be delivered, $73 \%$ of which will be single aisle aircraft [2]. Titanium production in the USA has been rising steadily to meet this increasing demand for new airframes and aeroengines. Large orders for new fuel efficient aircraft such as the B787, B777X, B737 MAX, A320NEO, A350XWB that use next generation engine architectures and light weight composite structures are being received [2]. Good compatibility between titanium and carbon fiber composites has led to increased use of titanium in new airframe structures containing larger amounts of composites [2].

While the aerospace sector continues to expand, numerous opportunities exist to increase titanium usage within the aerospace and other market sectors. These include developing new manufacturing processes to lower cost, improving performance of legacy and new titanium alloys to meet enhanced requirements for new applications, and developing computational materials models to reduce new alloy development cycle times and/or acceleration of process qualifications.

This paper reviews progress made during recent years in the United States. New alloys have been developed to meet enhanced component requirements, and new technologies, such as Additive Manufacturing (AM) have seen insertion opportunities in aerospace applications. Computational materials modeling tools are also being implemented to overcome challenges to the increased use of titanium.

\section{Additive Manufacturing}


Additive manufacturing (AM) provides an opportunity to design for intended component function with a high level of complexity and significantly reduced buy-to-fly ratio (purchased weight/fly away weight) [3]; however, it is not sufficient to just control part specific geometries and bulk compositions. The resulting component must have sufficient internal integrity, and repeatable metallurgical performance attributes to be fully qualified for service. In addition, AM must overcome high titanium powder costs, surface roughness, thermally induced residual stresses/distortions, costly inspection methods and process variability leading to component property scatter [4-5]. Several AM technologies are currently being used for titanium alloy manufacture in the United States.

Norsk Titanium recently began supplying the world's first FAA-approved aerospace-grade, structural titanium components. Boeing has implemented these Ti-6Al-4V parts, produced by the Rapid Plasma Deposition ${ }^{\mathrm{TM}}$ $\left(\mathrm{RPD}^{\mathrm{TM}}\right)$ wire-fed process in the 787-9 passenger floor galley diagonal fittings [6]. This RPD ${ }^{\mathrm{TM}}$ manufactured non-critical part was intentionally selected for the initial application (Figure 1). Material and component tests have been conducted to ensure the printed parts meet FAA requirements as well as Boeing specifications.

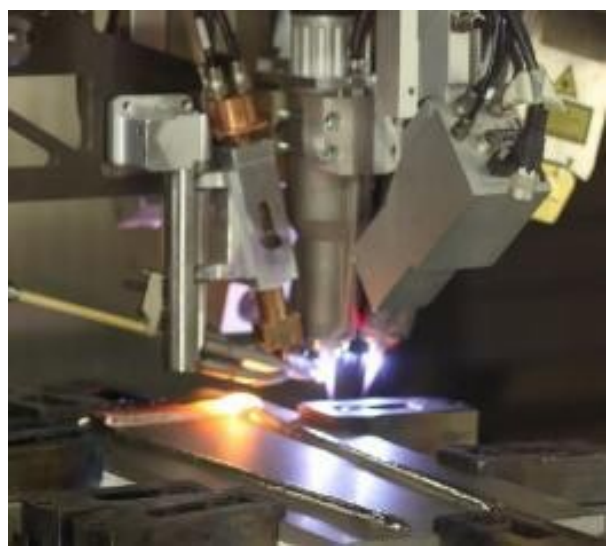

Figure 1. Part deposition in MERKE IVTM RPDTM Machine (Picture courtesy of Norsk Titanium)

Arconic has been incorporating the new technology into a comprehensive approach for highly sophisticated metallic products and processes, advancing technology for Additive Manufacturing in multiple processes to provide holistic solutions. In addition to direct manufacturing of components, the Ampliforge ${ }^{\mathrm{TM}}$ process combines additive manufacturing with the forging process to produce parts that meet the most stringent specification and integrity requirements while reducing costs for near-net geometries. The Electron Beam Direct Energy Deposition (EB-DED) process is capable of producing large aerospace components with high build rates. This is typically followed by hot isostatic pressing (hipping) to close and heal potential internal flaws [7]. Research by Lockheed Martin on EB-DED components shows that scatter in tensile properties is reduced, and inspectability is improved [8]. Effect of the deposition patterns has been studied at Lockheed Martin to reduce distinct microstructural banding for electron beam direct manufacturing of titanium (Figure 2). It was found that offsetting alternate layers appears most effective at improving ultrasonic inspection response across the preform. Cost impacts associated with staggered deposition patterns are minimal [9]. 


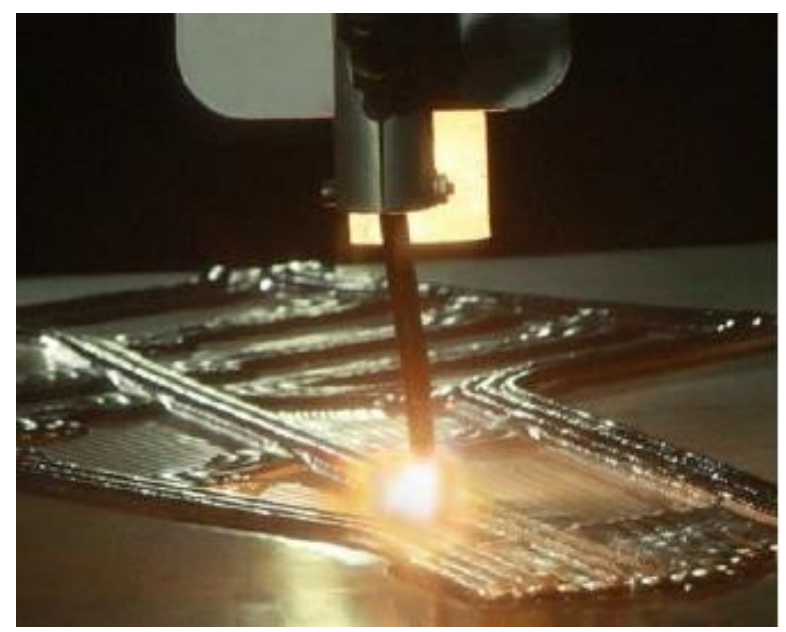

Figure 2. Wire Fed, Electron Beam - Direct Energy Deposition Process Producing Representative Ti-64 Fighter Component

(Picture courtesy of Lockheed Martin)

Studies on Ti-6Al-4V cylindrical single wall builds fabricated using electron beam directed energy AM have reported highly textured microstructures with $\{001\} \beta$ planes oriented to the principal machine axes [10]. Lowest strength was observed along the tensile axis aligned to either bead direction or normal to build plane, where favorable orientation of the preferred slip system enables easy dislocation motion. In addition, results showed that process interruptions did not affect material properties across the interface.

Research into establishing composition-microstructure-property-performance relationships led to exciting discrete (and integrated) advances. A recent DARPA program included significant efforts to understand and model the interrelationships between a wire-fed electron beam additive manufacturing process and the resulting static properties by considering the evolution of composition and microstructure. The results and the property model can be used to predict the yield strength of Ti-6Al-4V within $\sim 1 \%$ [11]. During this work it became necessary to develop a new tool to characterize the crystallographic orientation of titanium and its alloys over length scales of square decimeters, which subsequently drove the building of a spatially resolved acoustic spectroscopy (SRAS) system.

\section{New Alloy Development}

Ti-575, developed by TIMET, is an $\alpha+\beta$ alloy designed for improved strength and fatigue performance for engine discs and blades. Reports suggest a lack of dwell susceptibility in Ti-575, which may be due to the reduced macrozone size in billet product as well as the tri-modal microstructure in the SHTA condition [12]. Studies on TIMET's high ductility and damage tolerant alloy, Ti-407, are ongoing. Additional work on understanding prevalent deformation mechanisms in the alloy for improved fatigue performance compared to Ti-6Al-4V is progressing at several universities. These alloys are being evaluated by OEM's as potential replacements for traditional alloys such as Ti-6Al-4V in select air foil and rotor applications. 
Arconic-THOR ${ }^{\mathrm{TM}}$ was developed to target high temperature, structural aerospace applications. Arconic collaborated with Boeing and Honeywell under a United States Air Force funded Metals Affordability Initiative (MAI) project for focused maturation of Arconic-THOR ${ }^{\mathrm{TM}}$. Building upon the detailed technical investigations conducted on sub-scale systems to optimize material and processing, full-scale components were fabricated using production heat lots and manufacturing equipment. Manufacturing assessments and coupon/element testing were completed to demonstrate the product readiness. Statistically significant test data were generated and an aerospace material specification AMS6953 was recently granted for Arconic-THOR ${ }^{\mathrm{TM}}$ sheet production. Arconic-THOR ${ }^{\mathrm{TM}}$ can be produced as structural castings, and wrought products such as sheet, plate, foil, billet, rolled rings, forgings, and extrusions. The sheet product is formable (cold, hot, and superplastic forming), heat treatable, weldable using traditional methods. Successful product demonstrations of Arconic-THOR ${ }^{\text {TM }}$ are in direct support of insertion opportunities for legacy systems and next-generation hardware where extended operating temperature (increase of $200 \mathrm{oF}$ ) and improved oxidation resistance are required. This high temperature oxidation resistance capability bridges the gap between current state-of-the-art titanium alloys and heavier nickel superalloys. Key attributes of Arconic-THOR ${ }^{\mathrm{TM}}$ include 50\% lower weight than incumbent nickel superalloys for higher temperature applications, driving cost savings and fuel efficiency for customers. Superior oxidation resistant properties enable Arconic- THOR ${ }^{\mathrm{TM}}$ to operate at higher service temperatures than legacy alloys, offering the possibility of longer service life for those components that are life limited [13].

In addition to new alloy development, various studies have also been carried out to improve properties of existing titanium and titanium aluminide alloys. The effects of temperature and thermomechanical loading on the tensile strength and hardness were studied at Michigan State University for a rolled, low-cost metastable $\beta$ titanium alloy, Ti-13Cr-1Fe-3Al (wt.\%). Tensile tests performed at temperatures between $22^{\circ} \mathrm{C}$ and $410^{\circ} \mathrm{C}$ indicate that the ultimate tensile strength (UTS) decreased with increasing temperatures up to $300^{\circ} \mathrm{C}$. However, at $410^{\circ} \mathrm{C}$ the UTS was reported to be approximately $150 \%$ higher than the UTS at room temperature. It is believed that the formation of nanoscale $\omega$-phase precipitates, observed using transmission electron microscopy (TEM), impeded the movement of dislocations, which resulted in the exceptionally high UTS. Ductility was maintained, as the elongation-to-failure ( $\varepsilon f$ ) was $\sim 10 \%$ at $410^{\circ} \mathrm{C}[14]$.

Research and development of titanium based intermetallics has been progressing for over five decades. Although these alloys possess an attractive combination of high temperature properties along with low density, high manufacturing costs and low ductility have been a challenge for wider structural aerospace use. Case Western Reserve University recently studied the effects of thermomechanical processing, sample orientation, and load ratio on room- temperature fatigue crack growth and fracture behavior of a third-generation gamma titanium aluminide Ti-43.5Al-4Nb-1Mo-0.1B (TNM), currently used for low-pressure turbine airfoils. The CWRU work documented significant differences in microstructure, fracture behavior and fatigue properties for as-cast, cast+HIP, and forged conditions [15].

\section{Powder Processing}

A novel powder production technology using $\mathrm{Mg}$ to reduce $\mathrm{TiO} 2$ directly in a hydrogen atmosphere was discovered by Prof. Fang's group at University of Utah. Currently, $\mathrm{Mg}$ is used to react with titanium oxides in an inert atmosphere, resulting in Ti-powder with $>1 \mathrm{wt} \%$ the oxygen content, due to the inherent thermodynamic stability of Ti-O solid solutions as compared with $\mathrm{MgO}$ solutions. The Utah team reported that 
$\mathrm{H} 2$ can destabilize Ti-O solid solutions, thus enabling $\mathrm{Mg}$ to extract more oxygen out of Ti-O systems, and thereby producing Ti powder with very low oxygen content $(<0.1 \%)[16]$.

HSPT (Hydrogen Sintering and Phase Transformation) is a sintering process for manufacturing high performance near-net-shape Ti parts. HSPT sinters Ti powder compacts in a hydrogen atmosphere instead of high vacuum, as it is done conventionally. The presence of controlled $\mathrm{H} 2$ results in ultrafine microstructures in the as-sintererd state, while the conventional vacuum sintering process produces very coarse lamellar microstructure. The ultrafine, as-sintered microstructure of HSPT can then be heated to produce either globular or bi-modal microstructures, with equivalent mechanical properties that are standard for wrought Ti-6Al-4V. Results have shown that the fatigue endurance limit of Ti-6-4 produced by the HSPT process is far superior than that of conventional PM Ti, and equivalent to that of wrought Ti-6-4 [17].

\section{Advanced Manufacturing}

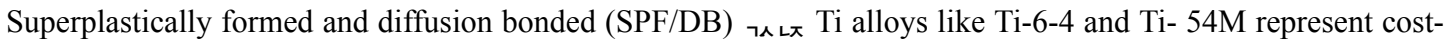
effective ways to manufacture complex shaped, internally stiffened, hollow metallic aerospace structures. Examples inlcude fan blades, nacelles, nozzles, and heat shields, as in Figure 3[18]. Development of these technologies continues for specific aerospace applications that enable improved performance of net generation aircraft.

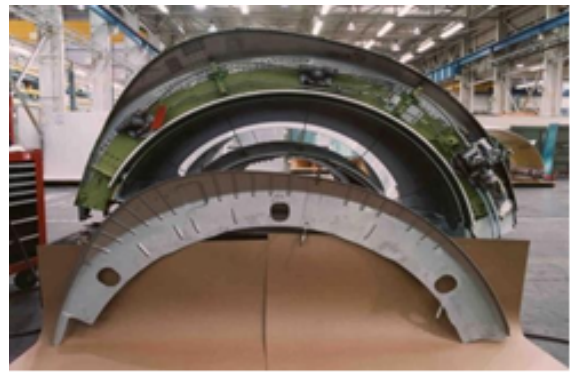

(a)

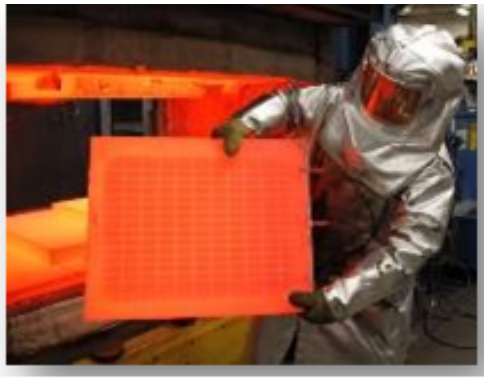

(b)

Figure 3. (a) 737-NG Thrust Reverser Heat Shield. Conventional design in the back; SPF design at front. (b) SPF/DB part with internally stiffened, hollow metallic structure. (Pictures courtesy of Boeing)

Titanium alloys are difficult to machine at high speeds, thus contributing to high product costs. Improvements in machining technology that address these challenges are essential for the growth of titanium in aerospace. Cryogenic machining of titanium has been studied using concentrated cooling (liquid nitrogen) at the cutting tool edge, which leads to higher heat dissipation and suppression of thermo-chemical reactivity between the cutting tool and workpiece. Lockheed Martin reported approximately 50\% improvement in material removal rate, $45 \%$ reduction in fabrication time, and $28 \%$ cost saving through the use of cryogenic machining 
technology, as shown in Figure 4 [19].

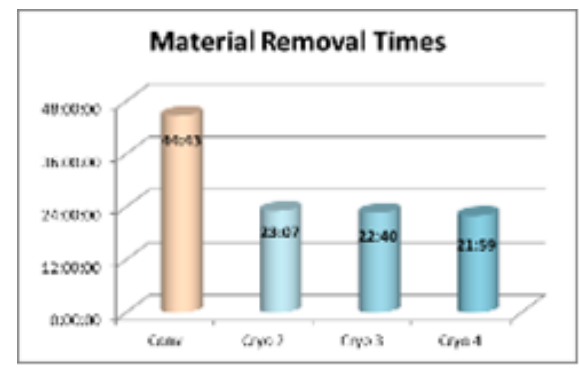

Figure 4. Material removal time by cryogenic machining trials (cryo2,3,4) vs conventional machining[19] (Picture courtesy of Lockheed Martin)

Ring rolling is a critical process for large diameter $(\sim 1200 \mathrm{~mm})$ and thin walled $(\sim 18 \mathrm{~mm})$ products; however, cracking and warping have been persistent issues affecting product quality and yield. Arconic has successfully mitigated these manufacturing challenges by optimizing the contour designs and utilizing special tools for forging and heat treatment processes. As result, the yield for the GR Ti6242 series parts has been improved from $66 \%$ to $97 \%$ [20].

A novel electro-chemical process that is environmentally friendly has been developed and patented by MetCon, LLC. This metal conditioning and pickling process replaces traditional full surface grinding, machining and acid pickling steps with a reduced yield loss electrochemical treatment. Traditional conditioning practices include grinding down to the bottom of the deepest cracks to avoid subsequent propagation during hot or cold working/processing steps (Figure 5). In contrast, the MetCon processes retains the majority of the surface bulk metal while opening surface cracks and blunting the crack tips, resulting in less overall material loss [21].

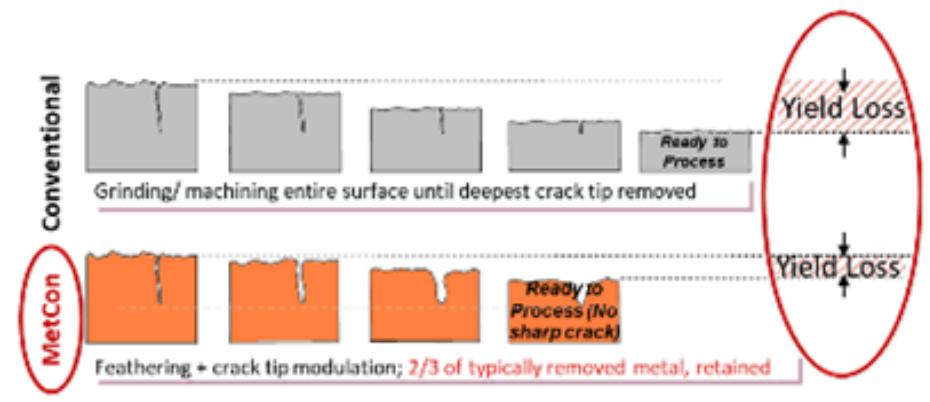

Figure 5. MetCon process retains $2 / 3$ of the metal lost during conventional conditioning (Picture courtesy of MetCon) 
Utilization of gamma titanium aluminide alloys in aerospace and other applications has placed significant demand on melters of these materials for products to be used in cast, wrought, and direct-machining applications. Over the past several years, Arconic Engineered Structures has developed a robust melting method using plasma cold-hearth melting technology, including the design and implementation of a new 3-torch system being utilized for production of Ti-48-2-2 cast bars. The 3-torch furnace is presently utilized for manufacturing machining stock for the LEAP low-pressure turbine blade [22].

\section{Computational Materials Engineering}

Performance, quality and cost requirements of titanium components drive the utilization of multi-disciplinary optimization methods that are well supported by Integrated computational materials modeling (ICME). Integration of models from across several engineering disciplines, such as design, structural analysis and manufacturing, can yield significant benefits in optimal materials, processes and component designs. Such an ICME framework has been demonstrated under the United States Air Force (USAF)-sponsored Foundational Engineering Problem (FEP) on optimizing aerospace components for location-specific properties that meet design intent [23]. The FEP developed model-based approaches to define manufacturing requirements to optimize residual stresses in rotor components to meet enhanced performance needs of next generation aeroengines.

In addition, Digital Thread efforts, supported by the US Department of Defense and focusing on integrating and analyzing digital representations of data to enhance acquisition and sustainment practices, are rapidly progressing. The USAF has sponsored several ICME programs that have successfully developed and validated microstructure and texture based fatigue models for dual phase titanium alloys [24]. The PW-9 program, investigated the evolution of microtexture and its effect on cold dwell fatigue behavior on Ti-6242 and Ti-64[25]. Specifically, this program successfully developed key tools to assess cogging process routes relative to microtexture, EBSD based methods to identify, classify and quantify microtextured regions (MTRs) and microstructure and microtexture based fatigue models for Ti-6-4 and Ti-6242. The goal of two other MAI programs, RR-12 and RR-13, was to build models to predict location-specific high and low cycle fatigue properties as a function of microstructure, texture and surface peening. These projects addressed the effect of manufacturing process and composition related variation on critical fatigue properties, observed in titanium components.

Collaborative University-Industry efforts to develop and validate accurate physics based models that utilize crystal plasticity finite element (CPFE) methods to predict crack initiation and growth in Titanium alloys is showing much promise. Researchers at Purdue University have been using CPFE based micromechanical modeling, that accounts for heterogeneous deformation resulting from microstructure and microtexture

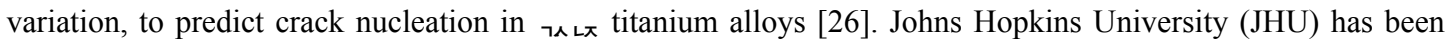
developing a multi-scaled parametrically homogenized constitutive modeling (PHCM) approach that explicitly represents microstructural descriptors (morphology and crystallography) in constitutive parameters that permit large scale coupon or component level analysis [27].

Design of titanium components continues to be based on empirically determined global minimum allowable properties, that results in conservative designs, adding to material, manufacturing and life cycle costs. Reducing the scatter in fatigue properties and incorporation of location-specific properties into component design are 
necessary to improve the affordability of titanium components. The previously mentioned ICME projects are enabling aircraft manufacturers and their suppliers to predict microstructural evolution (e.g., primary alpha size and volume fraction) as a function of process path. This has allowed OEM's and product suppliers to utilize linked process-structure-property models to define path and component location based process and material requirements.

Recently, NASA sponsored a study to define a future state by the year 2040 that would utilize integrated, multiscale modeling of materials and systems to accelerate the pace of innovation in future aerospace systems [28]. The NASA Vision 2040 report identified nine core technical areas for continued research and development efforts to enable an "ecosystem that impacts the supply chain to accelerate model-based concurrent design, and deployment of materials and systems throughout the product lifecycle for affordable, producible aerospace applications." Data informatics is a key element identified as being critical for the electronic capture of data from various supply chain sources, and extracting process-structure-property (PSP) relationships.

Deep learning models have enjoyed considerable success in fields such as computer vision, image and audio recognition, natural language processing, producing results comparable and even superior to human experts [29]. The ability of deep learning models to automatically learn important features in the data is a significant advantage over traditional modeling and image analytic approaches. Current applications of structural materials in the aerospace industry demand the highest quality control of material microstructure, especially for advanced rotational turbomachinery in aircraft engines to have the best-tailored material properties. Pratt \& Whitney $(\mathrm{P} \& \mathrm{~W})$ has recently developed deep convolutional neural networks to address fundamental challenges in materials engineering, such as the automated extraction of statistical information from dual phase titanium alloy microstructure images to support rapid engineering decision making, Figure 6. These models automatically learn critical features, without the need for manual specification and/or subjective and expensive image analysis.
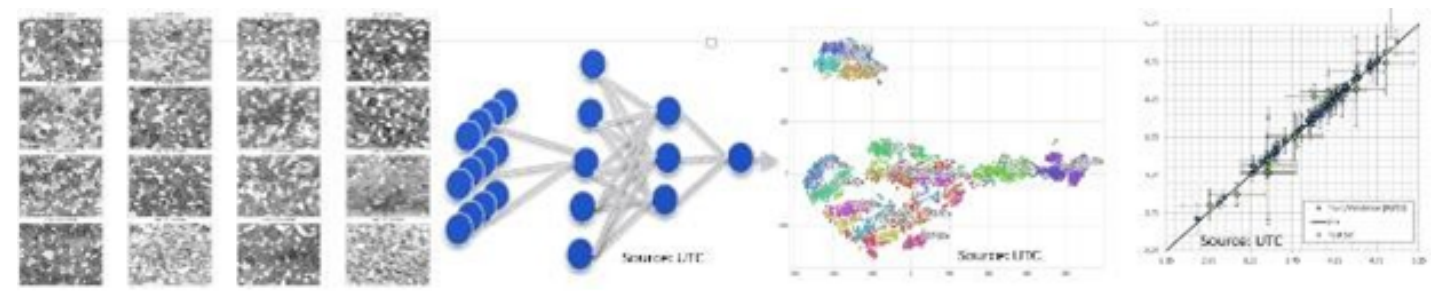

\section{Figure 6. Automated hierarchical image representations using deep learning methods. Figure shows an example of this approach using Ti-6Al-4V images to predict mechanical properties.}

Manufacturing process optimization using modeling and simulation tools is being implemented successfully by industry. Recently, Boeing developed and implemented a modeling simulation solution for linear friction welding of titanium for Ti-6Al-4V plate, bar, and forged stock, Figure 7. The manufacturing simulation tool was successfully developed, and sufficiently mature for engineering staff use [30]. 


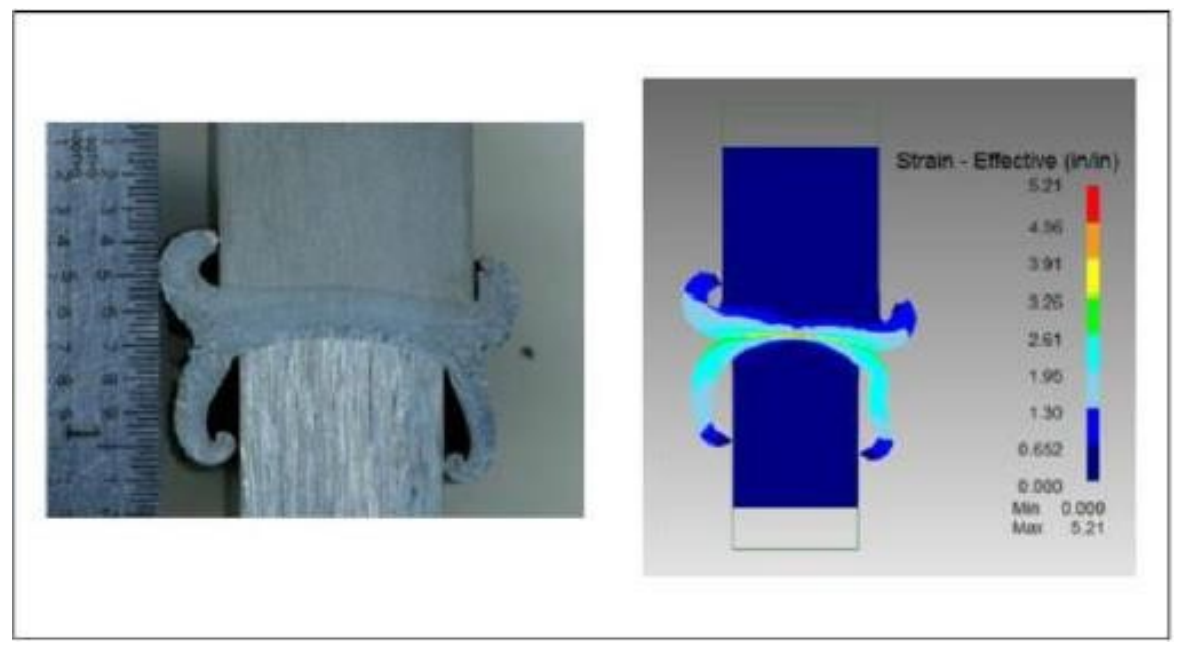

\section{Figure 7. Deformation modeling using DEFORM FEA for linear friction welding for aerospace components [30] (Picture courtesy of Boeing)}

Abnormal beta grain (ABG) growth resulting from beta heat treatment of forged of Ti-6Al- 4V-ELI is a localized microstructural phenomenon leading to very coarse beta grains. ABG is an industry-wide issue that has significant impact on material properties critical to aerospace applications. Model simulations of potential thermo-mechanical process paths, along with focused shop floor trials helped Arconic identify root cause of abnormal beta grain growth. Arconic subsequently developed an ABG indicator, based on modeling results as well as in-house open die forging and close die forging trials. This ABG indicator provides good predictions on the location and likelihood of $\mathrm{ABG}$ formation (Figure 8), leading to optimized process windows that mitigate ABG-prone forging conditions [31].

Recent work at AFRL on Ti-6Al-4V has reported that the occurrence of coarse grains may be due in part to the stabilization of a $\{001\}<110>$ texture during hot working that was further enhanced in intensity at the expense of other texture components during the early stages of $\beta$ annealing. This work suggests the best strategy to avoid $\mathrm{ABG}$ in $\beta$-annealed forgings is to control the strain and texture [32]. 


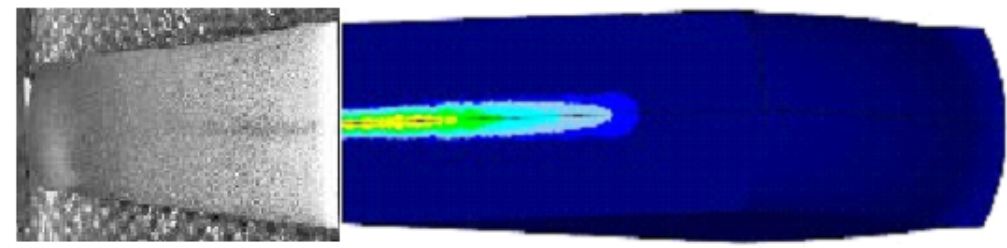

(a)

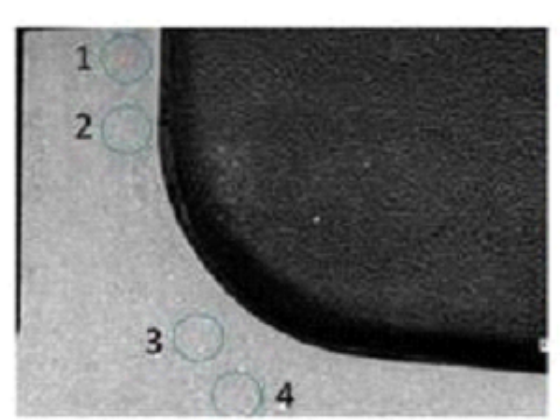

Measured regions of beta growth

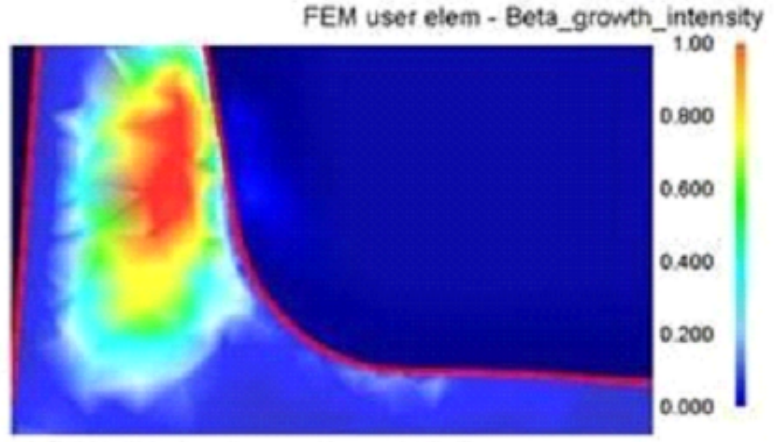

ABG indicator from the model: Significant growth

(b)

Figure 8. (a) Macrostructure showing abnormal beta grains (ABG) in the side press forged Ti6Al4V ELI billets and model predictions using ABG indicator; (b) A section in a complex- shaped part Macrostructure with ABG and model prediction using ABG indicator (Picture courtesy of Arconic)

\section{Non-Aerospace Applications}

Older concrete bridges in the United States were not built for today's standards. These bridges have inadequate steel reinforcement and were not designed for the current higher legal truck load demands. Replacement or rehabilitation is usually costly, and it is often more practical and cost effective to consider rehabilitation to extend the bridge life. The Perryman Company has been using titanium in the infrastructure market since 2012. Titanium near surface mounts (NSM) have been shown to increase the shear and flexural strength of reinforced concrete in bridge girders. The original research was performed at Oregon State University with full size beam testing to prove this technology. In NSM strengthening, grooves are cut into the concrete at a shallow depth to avoid the internal steel. Then a bar or strip is placed in the groove and held in place with epoxy. With titanium, the technique is unique because it includes 90 -degree anchorage hooks on both ends of the bar, resembling a staple. These anchorage hooks provide additional strength without being completely dependent upon the epoxy bond with the concrete (Figure 9). Titanium has high strength and good ductility along with complete corrosion resistance. It can survive in harsh environments with little or no concrete cover. In this application, titanium is actually more cost effective than the competing materials. This is a proven application and has been used on a 
number of bridges, with the state of Oregon leading the way. A design guide and an ASTM specification are available to assist design engineers [33].

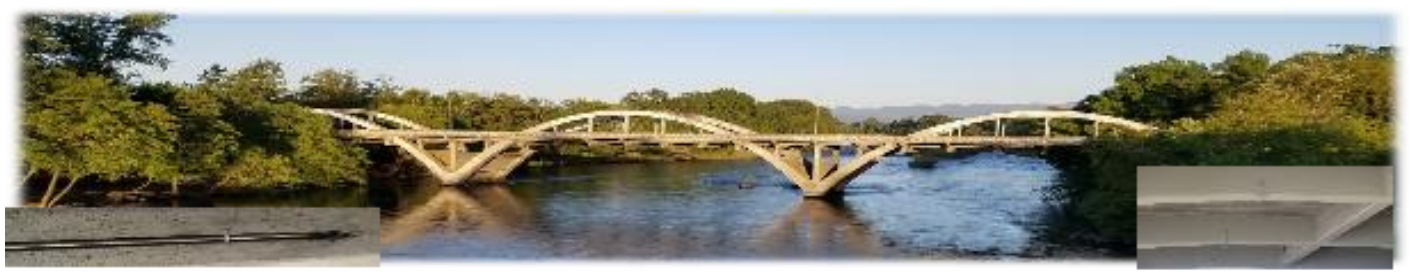

Figure 9. Rogue River Bridge with titanium bar strengthening. Built in 1931 in Grants Pass, OR. (Picture courtesy of Perryman Company)

\section{Summary}

Substantial progress has been made in the last several years in the US with regards to titanium technology, products, processing, and applications. Additive Manufacturing has been used much more widely to produce titanium components for use on commercial and military aircraft. The major US titanium manufacturers have been active in developing new titanium alloys, and a few high performance titanium alloys have been developed for higher temperature service and other applications. Computational modeling has been utilized extensively to optimize current production processes for titanium, and assist in developing new processes to further reduce cost and improve quality of these products. The Titanium Industry and academia are working closely together to address certain fundamental industry-wide issues, such as abnormal beta grain growth, need for low-cost titanium powders, and low or very high cycle fatigue behavior.

\section{$\underline{\text { Acknowledgements }}$}

The authors are extremely grateful to the following people who provided key information on titanium research and manufacturing. These include, Jim Cotton, Hank Phelps, Kayla Calvert, Dustin Bush, Melanie Chastka, Ernie Crist, Zhen Huang, Kurt Faller, Jill Adkins, Carl Boehlert, John Lewandowski, Zak Fang, Adam Pilchak, Peter Collins

\section{REFERENCES}

1. FAA Aerospace Forecast 2019-2039

2. ITA Executive Summary from Titanium USA 2018 conference

3. W. E. Frazier, MRS Bulletin, Col. 41, 10/2016

4. M. Qian, et al., Titanium Powder Metallurgy - Science, Technology and Applications, , Elsevier, Oxford, 2015

5. E. D. Herderick, JOM, 67, No. 3, 2015

6. H. Phelps, Personal Communication, Lockheed Martin Corporation, 2019

7. M. Chastka, "Arconic WDED Additive Manufacturing and the Ampliforge ${ }^{\mathrm{TM}}$ Process" - FIA/FIERF Forging Industry Technical Conference 2018 Long Beach https://www.forging.org/conference-presentations 
8. H. Phelps, Personal Communication, Lockheed Martin Corporation, 2019

9. H. Phelps, Personal Communication, Lockheed Martin Corporation, 2019

10. B.J. Hayes, et al., "Predicting tensile properties of Ti-6Al-4V produced via directed energy deposition", Acta Mater 133 (2017) 120-133

11. I. Ghamarian, et al., "A Constitutive Equation Relating Composition and Microstructure to Properties in Ti-6Al-4V: As Derived Using a Novel Integrated Computational Approach", Metal Trans A, Vol. 46A, 2015

12. K. Calvert, Timet, Personal Communication

13. F. Sun, et al, "Improved Oxidation Resistance in Titanium Alloy RTI-THORTM", AeroMat 2017

14. V. Khademi, et al, "The Elevated-Temperature Strength enhancement of a low-cost beta Titanium Alloy Through Thermomechanically-Induced Phase Transformation”, JOM, Issue 7, P71

15. M. Dahar, et al, "Evolution of fatigue crack growth and fracture behavior in gamma titanium aluminide Ti-43.5Al-4Nb-1Mo-0.1B (TNM) forgings", International Journal of Fatigue, 111(2018)54-69

16. Y. Zhang, et al, "Thermodynamic Destabilization of Ti-O Solid Solution by $\mathrm{H} 2$ and Deoxygenation of Ti Using Mg”, Journal of the American Chemical Society, 2016, 138: 6916-6919

17. Z. Zak Fang, et al, "Hydrogen Sintering of Titanium to Produce High Density Fine Grain Titanium Alloys", Advanced Eng. Materials, 14 (2012), p. 383-387

18. D. Sanders, Personal Communication, Boeing, 2019

19. J. Cotton, Personal Communication, Boeing, 2019

20. Zhen Huang, Personal Communication, Arconic, 2019

21. K. Faller, “ The Largest Cost Reduction Opportunity for Titanium Manufacturing in a Quarter Century: Electrochemical Conditioning and Finishing" 2015 ITA Conference

22. E. Crist, et al, "Advancement of Plasma Cold-Hearth Melting for Production of Gamma Titanium Aluminide Alloys within Arconic", TMS 2017, San Diego, CA, USA

23. V.Venaktesh, et. al., An ICME Framework for Incorporating Bulk Residual Stresses in Rotor Component Design, IMMI, 2018, https://doi.org/10.1007/s40192-018-0119-6

24. M. Glavicic, et. al., "Advanced Titanium Alloy Fatigue Modeling”, to be published in 2019 World Titanium Conference

25. V. Venkatesh, et. al., ICME of Microtexture Evolution in Dual Phase Titanium Alloys, 13th World Titanium Conference Proceedings, TMS, 2015

26. M. Sangid, K. Kapoor, Personal communication, Purdue University, 2019

27. S. Ghosh, Personal Communication, Johns Hopkins University, 2019

28. NASA 2040 Report, "A Roadmap for Integrated, Multiscale Modeling and Simulation of Materials and Systems", Contract NNC15BA06B http://www.sti.nasa.gov/, 2018

29. R. Noraas, et. al., "Structural Material Property Tailoring Using Deep Neural Networks", AIAA SCITECH 2019

30. A. Mann, et al, "An ICME Approach to Linear Friction Welding of Near-Net Shape Titanium Aerospace Structural Components", DMC 2017

31. D. Li, et al., Abnormal Beta Grain(ABG) Indicator Development, Arconic Studies, 2018

32. A.L. Pilchak, "Characterizing and Modeling the Precursors to Coarse Grain Formation during BetaAnnealing of Ti-6Al-4V", 2019 World Titanium Conference"

33. J. Adkins, "Designing with Titanium for Strengthening Concrete Bridges", Concrete Repair Bulletin, V. 31, No. 5, International Concrete Repair Institute, St. Paul, MN September/October 2018 